\title{
Water Impact Analysis for Aircraft over Sea
}

\author{
YAO Man \\ Xi'an University of Arts and Science \\ Xi'an 710065, China \\ 453244@qq.com
}

Keywords: aircraft over sea; water impact force; water-contact condition; overload

\begin{abstract}
The modern aircrafts' flying height is strictly limited by the conditions on the sea. Terrible conditions may lead overturning or dropping into the water to the aircraft. What's more, the structure of the aircraft may be broken by water-impact force. The affection of the water impact must be considered to promise the safety of the aircraft. This paper focuses on the water impact to a typical symmetrical aircraft flying above sea. The water impact force related to the trajectory angle and equivalent pitch angle is researched based on the water impact force model of a two-dimensional wedge. In addition, concerning with the limitation of the aircrafts' overload, the water-contact condition is proposed to avert attitude overturning and structure broken of the aircraft.
\end{abstract}

\section{Introduction}

Water impact is a significant factor for Aircrafts over sea. It cannot be ignored for this affection to the attitude of the aircraft. In fact, the flight velocity can be decomposed into the vertical velocity $V_{n}$ and the tangential velocity $V_{\tau}$ with respect to the tangent plane across the wave contact point. water-impact force is just the force due to vertical velocity $v_{n}$. Von Karman from American was the first researcher to define the water-impact force. He researched the impact-water phenomena during an aircraft landing on the water and proposed the rigid body impacting water theory in 1929 . The theory brought in a concept of added apparent mass to describe the water force on the contact surface of rigid body, and solved the impact problem by Momentum Conservation Law and the momentum theorem. Further researches done by others were most based on the theory ${ }^{[1,2,3]}$.

Generally, the research on the water-impact force always equaled the water-impacted body as a blunt rigid body with similar shape and volume moving along the vertical direction of a half space static flow, in which the fluid is ideal, incompressible and potential. Actually, the flow area, contact surface, free water surface can not be known exactly and the water-in time and depth is waited for a solution, all of which increase the complex of the problem. Over half a century, researchers were only able to calculate the force on the basis of a simplified problem. In all, there are three methods to calculate the water-impact force: the analytic method, the numerical method, and the experimental method. In this paper, analytic method, which is of clarified mechanism and clear meaning is applied to establish the water-impact force model and calculate.

\section{Water-Impact Force Model for a Two-Dimension Wedge}

The Water-Impact Force Model represented by Von Karman and Wagner describes a two-demission rigid wedge body, which drops vertically into a horizontal water surface, as shown in Fig. 1. Meanwhile, the following assumptions are made.

1) The water is incompressible and irrotational.

2) The cross section at bedewed surface of the wedge is equivalent to be a moving plate in infinite flow.

3) The width of the wedge socked by free water surface is $2 R$. 


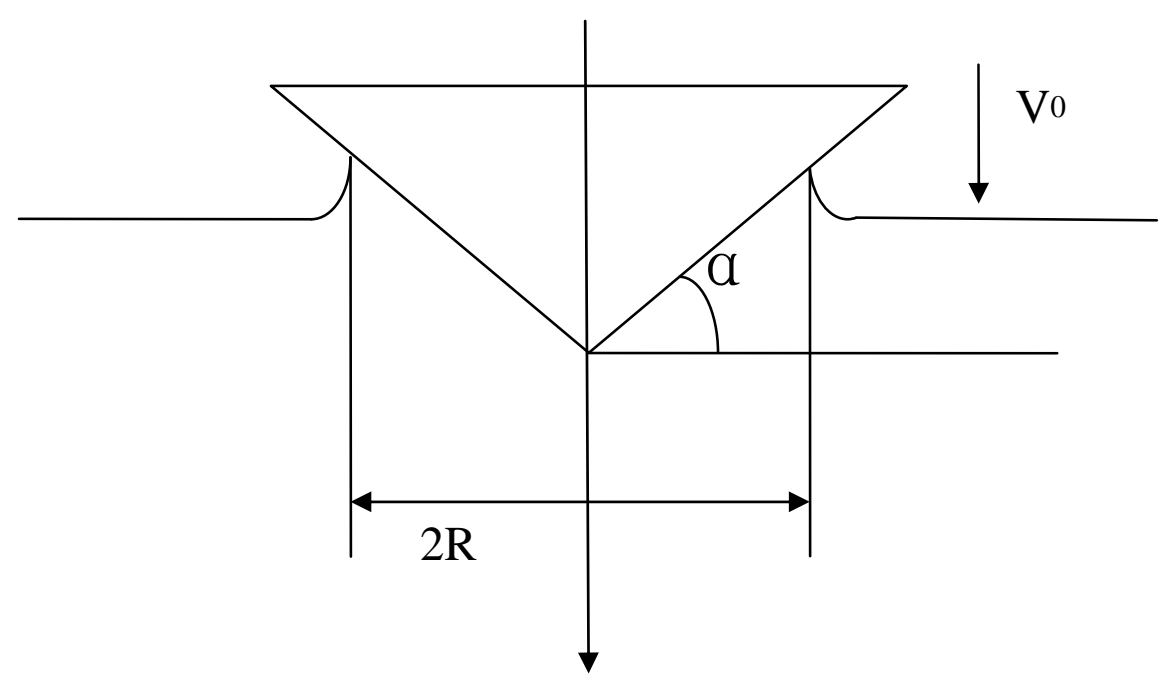

Figure 1. T Two-Dimension Wedge Body Impacting Water Model

The added apparent mass of the wedge under free water surface is

$$
m_{a}=\frac{1}{2} \pi \rho_{f} R^{2}(t)
$$

Where $\rho_{f}$ is the mass density of the fluid. Based on the Momentum Conservation Law, the following is obtained,

$$
M_{0} V_{0}=\left(M_{0}+m_{a}\right) V_{t},
$$

Where $M_{0}$ is the mass of the wedge body, $V_{0}$ is the vertical velocity relative to horizontal plane before the impact and $V_{t}$ after.

Derivate the momentum, substitute the momentum theorem $F=M \frac{d V}{d t}$, then we have

$$
F_{I P}=\frac{M_{0}}{M_{0}+m_{a}}\left(\pi \rho_{f} R \frac{d R}{d t}\right) V_{t} .
$$

According to Fig. 1 we have

$$
\frac{d R}{d t}=V_{t} \operatorname{ctg} \alpha,
$$

Where $\alpha$ is the oblique rising angle of wedge. Substitute Eq. 4 into Eq. 3, and the water impact force is given by[4,5]:

$$
F_{I P}=\pi \rho_{f} V_{t}^{2} R\left(1+\frac{\pi \rho_{f} R^{2}}{2 M_{0}}\right)^{-1} \operatorname{ctg} \alpha .
$$

\section{Water-Impact Force Model for an Aircraft Over Sea}

The Water-Impact Force Model in Eq. 5 reflects the effect of oblique rising angle of wedge on the force without taking water-in attitude into account. Therefore the model needs to be improved for closing to reality further with assumptions as follows. 
1) The undersurface of aircraft touched with water is equal to a wedge with small oblique rising angle, the socked length is $L$, the socked width by free surface is $b$, and the equivalent oblique rising angle is $\alpha_{0}$.

2) The aircraft mass is $M$, and the vertical velocity with respect to the tangent plane across the wave contact point is $V_{e}$.

3) The angle between aircraft and its wave-contact tangent plane is due to the flight attitude, define the angle between aircraft body axis $O x_{1}$ and the tangent plane is equivalent pitch angle $\phi^{\prime}$.

4) The actual bedewed area of aircraft undersurface is $S_{a}$.

5) The mass density of sea water is $\rho_{s}$.

Angles defined above to describe the water-impact condition are shown in Fig. 2.

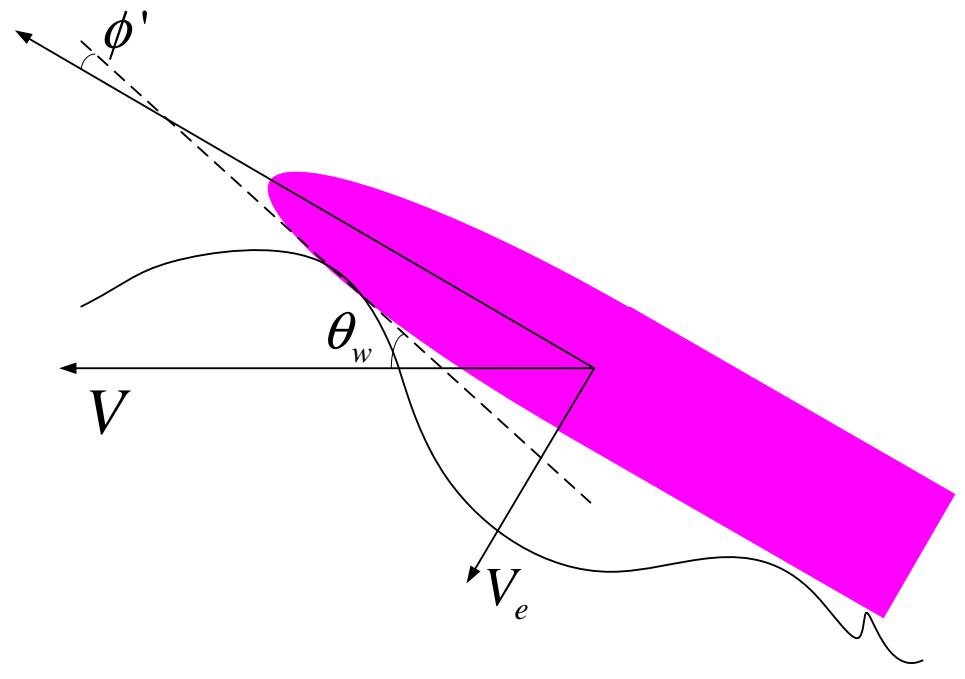

Figure 2. $\quad$ Angles of Aircraft over Sea Contacted by Waves

Based on Eq. 5, the Water-Impact Force Model involved with flight attitude is obtained by

$T=\frac{1}{2} \pi \rho_{s} V_{e}^{2} S_{a}\left(1+\frac{\pi \rho_{s} S_{a}^{2} \cos ^{2} \alpha}{8 M}\right)^{-1} \cos \alpha \operatorname{ctg} \alpha$.

Where $\alpha$ is the sum of two angles, one of which is the angle of equivalent oblique rising angle, $\alpha_{0}$, and the other is the attached angle due to flight attitude, $\alpha_{0}^{\prime}$.

$$
\alpha=\alpha_{0}+\alpha_{0}{ }^{\prime}=\alpha_{0}+\phi^{\prime}
$$

\section{Simulation and Analysis}

In this section, water-impact force under different conditions such as flight attitudes is calculated with model in Eq. 6. The conditions are as follows.

1) The aircraft is flying along the horizontal plane with a flight height of $2.5 \mathrm{~m}$ and flight velocity of $100 \mathrm{~m} / \mathrm{s}$.

2) The sea waves are acting like a series of sine waves, the amplitude of which are respectively 2.6m, $2.8 \mathrm{~m}$ and $3 \mathrm{~m}$.

3) Corresponding to different wave height, the trajectory angle $\theta_{w}$,which is the angle between flight velocity direction and wave-contact tangent plane, are respectively $-1.2^{\circ},-1.8^{\circ}$ and $-2.3^{\circ}$.

4) The equivalent pitch angle $\phi^{\prime}$ is $0^{\circ} \sim 5^{\circ}$.

5) Corresponding to different wave height, the actual bedewed area of aircraft undersurface $S_{a}$ are $0.76 \mathrm{~m}^{2}, \quad 1.35 \mathrm{~m}^{2}$ and $1.89 \mathrm{~m}^{2}$. 
6) The equivalent oblique rising angle is $\alpha_{0}=10^{\circ}$.

Use Eq. 6 to calculate the water-impact force under different conditions of wave and flight attitudes given above. Parts of the simulation results are shown in Tab. 1.

Table 1 The Water-Impact Force under Different Trajectory Angles and Flight Attitudes

\begin{tabular}{|c|c|c|c|c|c|}
\hline \multicolumn{2}{|c|}{ Trajectory Angle -2.3 deg } & \multicolumn{2}{c|}{ Trajectory Angle -1.8 deg } & \multicolumn{2}{c|}{ Trajectory Angle-1.2 deg } \\
\hline $\begin{array}{c}\text { Equivalent } \\
\text { Pitch Angle } \\
(\text { deg) }\end{array}$ & $\begin{array}{c}\text { Equivalent } \\
\text { Pitch Angle } \\
(\text { deg })\end{array}$ & $\begin{array}{c}\text { Water-Impact } \\
\text { Overload } \\
(\boldsymbol{g})\end{array}$ & $\begin{array}{c}\text { Water-Impact } \\
\text { Overload } \\
(\boldsymbol{g})\end{array}$ & $\begin{array}{c}\text { Equivalent } \\
\text { Pitch Angle } \\
(\text { deg })\end{array}$ & $\begin{array}{c}\text { Water-Impact } \\
\text { Overload } \\
(\boldsymbol{g})\end{array}$ \\
\hline 5.0 & 5.0 & 3.6 & 5.1 & 5.0 & 1.6 \\
\hline 3.0 & 3.0 & 4.3 & 6.07 & 3.0 & 1.9 \\
\hline 2.5 & 2.5 & 5.2 & 7.3 & 2.5 & 2.3 \\
\hline 2.0 & 2.0 & 6.1 & 8.5 & 2.0 & 2.7 \\
\hline 1.5 & 1.5 & 6.9 & 9.7 & 1.5 & 3.1 \\
\hline 1.0 & 1.0 & 7.6 & 0.6 & 1.0 & 3.4 \\
\hline
\end{tabular}

\section{Conclusion}

By analyzing the simulation results in Tab. 1, we've got some conclusions about the water-impact force changing as trajectory angle and equivalent pitch angle, which are shown as follows.

1) The trajectory angle is the most important factor to affect the water-impact force, to which the overload is in proportional when the angle is less than $10 \mathrm{deg}$. The reason is the velocity $V_{e}$ is directly related to trajectory angle. The bigger the angle is, the bigger $V_{e}$ is and thus the bigger the overload is.

2) The equivalent pitch angle has a smaller effect on water-impact force, and the overload is in inverse proportional to the absolute value of the angle.

3) When the aircraft impacted by water below 4th sea state(the sea wave height is between $2.5 \mathrm{~m}$ and $3 \mathrm{~m}$ ), it can bear water-impact overload less than $6 \mathrm{~g}$ without attitude overturning and structure broken. According to Tab. 1, the equivalent pitch angle should be bigger than 3 deg to keep the aircraft safe when the flight height is less than $2.5 \mathrm{~m}$.

4) Furthermore, the safety of aircraft flying over sea should be realized based on the consideration to flight mission and control system capability, and choosing proper trajectory angle, equivalent pitch angle and flight height.

\section{References}

[1] SCOTT W B. Airbreathing hyperSoar would 'bounce'on upper atmosphere[J]. Aviation Week \& Space Tech2 nology , 1998(7)

[2] Wang Zhidong, Zhu Renqing. Hydrodynamic Characteristics of 2-Dimensional Hydrofoil Moving near to Free-water Surface[J]. Ship Engineering,2004,26(3) (in Chinese)

[3] Von Karman T. The impact on seaplane floats during landing. National Advisory Committee for Awronautics,1929,321:145-162

[4] Wagner H. Phenomena associated with ioacts and sliding on liquid surfaces. National Advisory Committee for Aeronautics, 1932, 1366: 112-123

[5] Zhu Minhu. Hydrodynamic Force Calculation of a Slipping Boat. Shipping Technology Home and Abroad,2004(3) (in Chinese) 